\title{
Indoor precise positioning algorithm using 60GHz pulse based on compressive sensing
}

\author{
Xueyan $\mathrm{Han}^{\mathrm{a}, \mathrm{b}}$, Jingjing Wang ${ }^{\mathrm{a}, \mathrm{b}, *}$, Wei Shi ${ }^{\mathrm{a}, *}$, Quna Niu ${ }^{\mathrm{a}, \mathrm{c}}$, Lingwei $\mathrm{Xu}^{\mathrm{a}}$ \\ ${ }^{a}$ School of Information Science and Technology, Qingdao University of Science and Technology, Qingdao, Shandong Province, China. \\ ${ }^{b}$ Key Laboratory of Universal Wireless Communications (Beijing University of Posts and Telecommunications), Ministry of \\ Education, P. R. China.
}

'Shandong provincial Key Laboratory of Computer Network, Jinan, Shandong Province, China.

\begin{abstract}
Aiming at the existing defect of poor positioning accuracy in NLOS (Non Line of Sight) environment for most of the common indoor positioning algorithms, this paper proposes a precise indoor positioning algorithm using $60 \mathrm{GHz}$ pulse based on compressed sensing. The proposed algorithm converts the location of the target nodes in the area to be located into a sparse vector and designs the over-completed dictionary using TOA (Time of Arrival)-based ranging, then takes advantage of the $l_{1}$-minimization to reconstruct the location of the target nodes. The algorithm divides the positioning process into coarse positioning and fine positioning, and introduces the reference node selection mechanism in fine positioning. The algorithm not only can achieve the positioning of single target, but also achieve the positioning of multiple targets. Through the theoretical analysis and experiment simulation results, we can conclude that the proposed algorithm using $60 \mathrm{GHz}$ pulse can achieve precise indoor positioning in NLOS environment and centimeter-level positioning precision can be obtained compared with TOA based $60 \mathrm{GHz}$ geometric positioning algorithm. (c)2016 All rights reserved.
\end{abstract}

Keywords: 60GHz pulse, NLOS, compressed sensing, positioning.

\section{Introduction}

Positioning technologies are in great demand in many applications such as navigation, production management and internet of things, especially the positioning accuracy which is demanded increasingly. However, the existing variety of indoor positioning technologies exist some shortcomings, for instance, low positioning accuracy and high complexity due to the effects of the multipath, NLOS propagation and the other factors in

\footnotetext{
${ }^{*}$ Corresponding author

Email addresses: 564684119@qq.com (Xueyan Han), kathy1003@163.com (Jingjing Wang), shiwei6670595@126.com (Wei Shi), niuqiuna@sdu.edu.cn (Qiuna Niu), 13210120837@163.com (Lingwei Xu)
} 
NLOS environment and unable to meet the growing demand for high-precision of indoor positioning, thus it is urgently to seek new technologies and new theoretical solutions to achieve high accuracy indoor positioning in NLOS environment.

Since 2000, many country governments allocated continuous unlicensed communication band in the vicinity of $60 \mathrm{GHz}$ band [17]. $60 \mathrm{GHz}$ pulse communication system provides a license-free spectrum up to $7 \mathrm{GHz}$ used to communication compared with $2.4 \mathrm{GHz}$ and $5 \mathrm{GHz}$ communication systems which are common applied to short-range communication at present. Its abundant bandwidth resources laid the basis of the realization of high-speed wireless transmission in Gbps-level. Moreover, the spectrum is completely free. Therefore, $60 \mathrm{GHz}$ pulse communication technology has received increasing attention and becomes one of the most potential wireless communications technology in future under the inevitable trends of the wireless communication spectrum resources are increasingly strained and the data transfer rate becomes higher and higher in current [23, 24].

The duration of $60 \mathrm{GHz}$ pulse is extremely short, usually about hundreds of picoseconds or shorter, which is far less than the delay of the multipath propagation. For this reason, the multipath signal at the receiving end can be separated effectively and a high time resolution and multipath resolution can be gained. These qualities make the $60 \mathrm{GHz}$ pulse can achieve centimeter-level even millimeter-level ranging and positioning accuracy which supplies an effective way of design and implement high-precision indoor positioning systems. In recent years, many foreign academic communities and enterprise alliances were opened for the research of the $60 \mathrm{GHz}$ pulse wireless communication technology. But the research is mainly focused on the design of transceiver [16, 18, 21], the establishment of channel model [2, 9], the standards the physical layer and MAC layer of communication [10], the transceiver antenna beam shaping [12, 13, 26] and so on. Currently, the research of the $60 \mathrm{GHz}$ pulse positioning is still relatively deficient, and lack of an integral theoretical system. $60 \mathrm{GHz}$ pulse can achieve accurate positioning results in line-of-sight (LOS) environment depended on its high time resolution and multipath resolution. [20] completed the design and simulation of high-precision $60 \mathrm{GHz}$ pulse positioning system respectively from presenting a pulse waveform suitable for $60 \mathrm{GHz}$ pulse positioning, the process of pulse transmitting, channel transmission model, pulse receiving and positioning algorithm. It used the geometrical location algorithm based on TOA and least squares to complete the process of $60 \mathrm{GHz}$ pulse ranging and positioning process and can reach millimeter-level ranging accuracy and centimeter-level positioning accuracy in LOS channel. [15] designed a 40nm CMOS receiver and applied it to $60 \mathrm{GHz}$ pulse positioning system. It avoided the significant power penalty caused by high-bandwidth and achieved a millimeter-level positioning accuracy within the range of $4 \mathrm{~m}$. However, the $60 \mathrm{GHz}$ pulse precise positioning still exists many problems and challenges. The large signal propagation attenuation, difficulty to penetrate obstructions and the relatively weak multipath effect make the $60 \mathrm{GHz}$ pulse precise ranging and positioning in NLOS extremely difficult. At present, only a small amount of literature realized $60 \mathrm{GHz}$ pulse indoor precise positioning in NLOS environment.

[11] proposed MISO system based on TDOA (Time difference of Arrival). Unlike the conventional TDOA systems, the sending terminal can transmit two identical signals at the same time using two antennas and the receiving terminal can extract TDOA from the interference spectrum of the two signals to realize communication and positioning. In addition, it investigated the influence of NLOS on TDOA and positioning accuracy in different situations. [19] presented a fingerprinting based localization algorithm for $60 \mathrm{GHz}$ impulse radio positioning in NLOS. This algorithm made full use of the negative factors such as multipath and NLOS to construct the fingerprint database, and then measured the parameters of the received signal and used a matching algorithm to determine which group the received data belongs to and then used the information to estimate the position of the target node. The method can be applied to areas in which the multipath effects and NLOS propagation is more serious. It had a relatively higher accuracy compared with other targeting methods. But the disadvantage is the computational complexity and the poor adaptability to the environments. This shows that the existing $60 \mathrm{GHz}$ pulse indoor precise positioning algorithms have deficiency of low positioning accuracy, high complexity and so on. Thus, it is urgently to solve the problem of $60 \mathrm{GHz}$ pulse indoor precise location in NLOS. 
Compressive sensing (CS) is a novel theory of message acquisition proposed in recent years that can reconstruct a sparse or compressible signal precisely use a small number of samples [4, 7]. For the target node locations are sparse compared with the whole area to be located which satisfied the application conditions of CS, we can utilize signal reconstruction algorithm to realize the target location. In recent years, there already exist many documents applied CS to target location question and achieved reasonably good positioning results [8, 14, 22].

Based on the requirements of indoor positioning accuracy in NLOS and the characteristics of $60 \mathrm{GHz}$ pulse, combined with the emerging CS theory, this paper presents a new precise indoor positioning algorithm using $60 \mathrm{GHz}$ pulse based on CS which achieves the target node locations using signal reconstruction algorithm. Two positioning process [25] and the selection mechanism of reference stations are presented which reduce the calculation and the complexity of positioning compared with fingerprinting based localization algorithm. The centimeter-level positioning accuracy is achieved in NLOS.

The rest of the paper is organized as follows: Section 2 describes the $60 \mathrm{GHz}$ pulse positioning system model; Section 3 provides a detailed introduction of the localization algorithm in this paper; Section 4 reports our simulation results of single target localization and multi-target localization, analyzes it's positioning performance and compares it with geometric positioning algorithm based on TOA in positioning accuracy and Section 5 makes a conclusions of this study.

\section{The mathematical model of positioning system using 60GHz pulse based on CS}

\subsection{The principle of positioning based on $C S$}

For the position of each target node at any time in the area to be located is unique in the process of positioning, the position of the target node is relatively sparse compared with the entire area. This article converts the location of the $K$ targets in the area to be located into a $K$-sparse column vector $X=\left[x_{1}, x_{2}, \ldots, x_{N}\right]^{T}$, where $x_{n}=0$ or $1(1 \leq n \leq N)$, when there is a target node in the $n$-th grid $x_{n}=1$, otherwise $x_{n}=0$.

CS theory indicates that: If the signal $X \in R^{N}$ is $K$-sparse, by using the measurement matrix $\Phi \in$ $R^{M \times N}(M \ll N)$ to make a linear transformation of the signal, the linear projection of the signal $X$ under the observation of measurement matrix $\Phi$ can be obtained, namely: $Y=\Phi \mathrm{X}$. Candes et al. pointed out that [3], if the number of measurement $M$ met:

$$
M=O(K \log (N / K)), \quad M \ll N .
$$

Then the signal can be reconstructed in high probability by solving $l_{0}$-minimization problem:

$$
\hat{\mathrm{X}}=\underset{\mathrm{X} \in R^{N}}{\arg \min }\|\mathrm{X}\|_{0} \text { s.t. } Y=\Phi \mathrm{X} .
$$

However, the $l_{0}$-minimization is a non-deterministic polynomial problem [5]. Chen, Donoho and Saunders pointed out that it can be solved more simply by $l_{1}$-minimization to obtain the same result [6]. Therefore, the mathematical model of the target node localization problem can be expressed as the Equation (2.3):

$$
\hat{X}=\underset{X \in R^{N}}{\arg \min }\|X\|_{l_{1}} \text { s.t. } Y=\Phi X .
$$

Therefore, the problem of locating the $K$ target node in the area to be located is transformed into the problem that taking advantage of the measurement column vector $Y$, the measurement matrix $\Phi$ and the k-sparse signal $X \in R^{N}$ to reconstruct the location of the $K$ non-zero value in the sparse vector. Measurement column vector $Y=\left[y_{1}, y_{2}, \ldots, y_{M}\right]^{T}$, where, $y_{m}$ is the real distance between the target node and the $m$-th reference station based on TOA measured by transmitting $60 \mathrm{GHz}$ pulse signal at each target node, transporting by IEEE802.15.3c channel and receiving signal at the $m$-th reference station. 
After the sparse vector $\hat{X}$ is reconstructed through the reconstruction algorithm, the non-zero value of the sparse vector $\hat{X}$ is corresponded to the position of the target node, then the localization of the target node is accomplished.

\subsection{The design of the measurement matrix}

The method of construct measurement matrix $\Phi \in R^{M \times N}(M \ll N)$ : The elements $\varphi_{m, n}$ in measurement matrix $\Phi$ is the actual distance between the $n$-th grid node and the $m$-th reference station. At first, by transmitting $60 \mathrm{GHz}$ pulse signals at the $n$-th grid node and receiving signal at the $m$-th reference station we can obtain the propagation delay $\tau_{m, n}$ based on TOA, then calculating the distance on basis of $d_{m, n}=c * \tau_{m, n}$, we get $\varphi_{m, n}$ as shown in Equation (2.4):

$$
\varphi_{m, n}=d_{m, n}, 1 \leq m \leq M, 1 \leq n \leq N .
$$

The measurement matrix can be expressed as Equation 2.5):

$$
\Phi=\left[\begin{array}{cccc}
d_{1,1} & d_{1,2} & \cdots & d_{1, N} \\
d_{2,1} & d_{2,2} & \cdots & d_{2, N} \\
\vdots & \vdots & \ddots & \vdots \\
d_{M, 1} & d_{M, 2} & \cdots & d_{M, N}
\end{array}\right]
$$

Defining the sensing matrix $A=\Phi \Psi$. Since the signal $X$ is k-sparse, we select a $N \times N$ unit matrix as the orthogonal basis $\Psi$. Candes and Tao presented and proved in [3] that a sufficient condition for reconstructing a sparse signal successfully by $l_{1}$-minimization in CS theory was that the sensing matrix $A=\Phi \Psi$ obeys restricted isometry property ( RIP ) in CS theory. [1] showed that if the measurement matrix $\Phi$ and the orthogonal basis $\Psi$ is not related, the sensing matrix $A$ obeys RIP in high probability. Therefore, we make an orthogonal operation to the measurement column vector as follows:

Make $Z=T Y$, define $T=Q A^{\dagger}$, where $Q=\operatorname{orth}\left(A^{T}\right)^{T}$ and $\operatorname{orth}(A)$ represents an orthonormal operation for the matrix $A . A^{\dagger}$ is the pseudo-inverse matrix of $A$. Therefore, using the $l_{1}$-minimization the localization problem can be described as Equation 2.6) :

$$
\hat{X}=\underset{X \in R^{N}}{\arg \min }\|X\|_{l_{1}} \text { s.t. } Z=Q X+\varepsilon 1,
$$

Where $\varepsilon=T \varepsilon 1, \varepsilon 1$ is acceptable margin of error.

\section{The design of $60 \mathrm{GHz}$ pulse positioning algorithm based on CS}

\subsection{Analysis of the reconstruction algorithm}

In this paper, we use $l_{1}$-minimization to reconstruct the sparse vector. In order to apply the reconstruction algorithm better to $60 \mathrm{GHz}$ pulse precise indoor positioning system, this paper studies the performance of the reconstruction algorithm in the first place, mainly studies the influence of the signal length $N$, the number of observations $M$ and the sparsity level of $K$ to the reconstruction algorithm. The algorithm in this paper utilizes CS to achieve the localization of the target node based on fingerprinting. In the positioning process, the signal length $N$ corresponds to the number of grid node, the number of observations $M$ corresponds to the number of the reference station, the sparsity level of $K$ corresponds to the number of target node, and the probability of the signal to be exactly reconstructed corresponds to the probability of accurate positioning.

(1) We assume that the signal length $N=125$, the number of target node $K=1,2,4$ and 6 , respectively, the number of reference station $M$ varies from 0 to 125 at a step of 5 . Each set of parameters run 10000 times, then plot the statistical results of the 1000 repeated simulations in Fig. 1. We find from the simulation results that the probability of the signal to be reconstructed accurately increases as $M$ increases when $K=1$, and the number of the reference station $M$ required increases as $K$ increases when the signal is reconstruct accurately. 


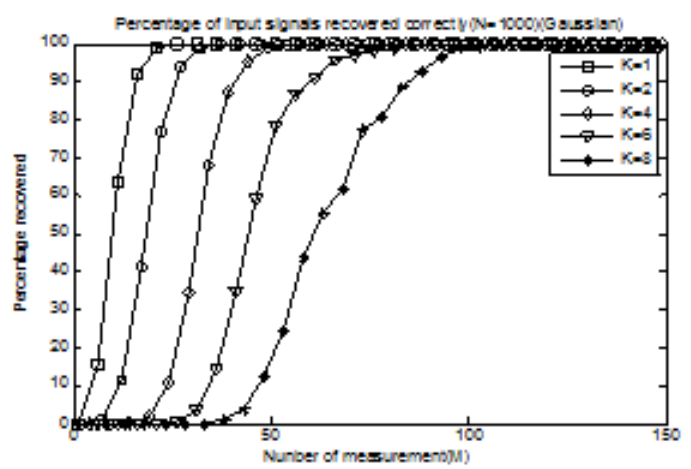

Figure 1: The relationship between the number of measurement $M$ and the probability of accurate reconstruction under different $K$-values.

(2) We assume that the length of the signal $N$ varies from 0 to 2000 at a step of 100 , the number of reference station $M=10,20,30,40$ and 50, respectively. Simulate and compare the reconstruction algorithm performance when the target node $K=1$ and 3. Each set of parameters run 10000 times, then plot the statistical results of the 1000 repeated simulations in Fig. 2. We observe from the simulation results that, when $K$ is a constant, the number of the reference station $M$ required increase as $N$ increase when the signal is reconstruct accurately.

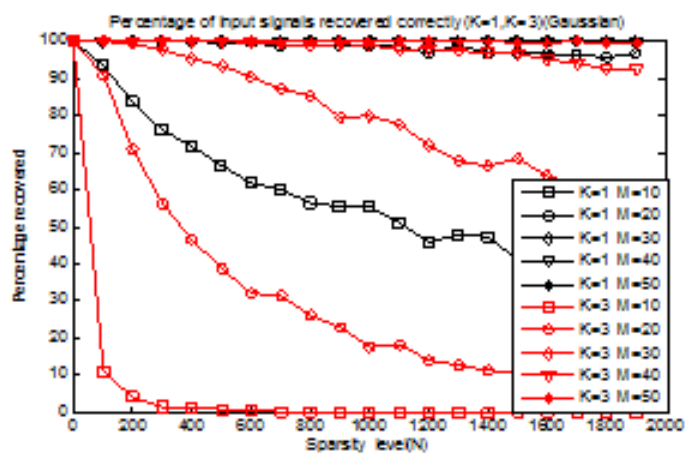

Figure 2: The relationship between the signal length $N$ and the probability of accurate reconstruction when $K=1$ and $K=3$.

Through the analysis of the reconstruction algorithm, the larger the area to be located and the finer of the meshing, the larger the number of grid node will be, and also the number of the reference station required. However, in the actual positioning process, it is unrealistic to set up too many reference stations in the located area due to the restriction of the environmental conditions and costs. To resolve this conflict, this paper divides the positioning process into coarse positioning and fine positioning, that is, complete the localization through twice reconstruction. During the second positioning process, it is likely to locate the target to the neighboring grid for the similarity of their measured data caused by part of reference stations are far away from the target node and the impact of measurement error. Thus, we present the reference station selection mechanism in fine positioning in order to get rid of some remote reference station. These measures will increase the positioning accuracy and reduce the computational complexity to a large extent.

Of course, when area to be located is changed or the positioning accuracy is altered, we can choose one time positioning or more times of iterative positioning according to the actual cases to meet the actual needs of the positioning accuracy. This paper mainly introduces the twice positioning process through single target positioning and multiple targets positioning.

\subsection{The single target positioning using 60GHz pulse}

The positioning process is divided to two phase: the offline stage and the online stage. The specific steps of the algorithm are as follows: 


\subsubsection{The offline stage}

The preparations before positioning is done during the offline stage, primarily to establish over-complete dictionary $\Phi$ preparing for the online stage. It is divided into two steps:

(1) Divide the three-dimensional area to be located into grid node.

Assume that, the three-dimensional area to be located is a $L \times L \times L$ square area, where $L$ is the side length of the square area. Firstly, the positioning computing center divided the three-dimensional area to be located into $N_{1}=N_{1 x} \times N_{1 y} \times N_{1 z}$ large grid node. $L 1$ is the side length of large grid. $N_{1 x}, N_{1 y}, N_{1 z}$ represent the number of the large grid node in the area to be located in $x, y, z$ directions respectively. The position of the $n 1$-th large grid node can be expressed as:

$$
P_{n 1}=P_{i, j, k}=\left(x_{i}, y_{j}, z_{z}\right), i=1, \ldots, N_{1 x}, j=1, \ldots, N_{1 y}, k=1, \ldots, N_{1 z},
$$

where $i, j, k$ represent the order of the n1-th large grid in $x, y, z$ directions respectively.

Secondly, the positioning computing center divided the large grid into $N_{2}=N_{2 x} \times N_{2 y} \times N_{2 z}$ small grid node. $L 2$ is the side length of small grid. $N_{2 x}, N_{2 y}, N_{2 z}$ represent the number of the small grid node in the large grid in $x, y, z$ directions respectively. The position of the $n 2$-th small grid node can be expressed as:

$$
P_{n 2}=p_{l, p, q}=\left(x_{l}, y_{p}, z_{q}\right), l=1, \ldots, N_{2 x}, p=1, \ldots, N_{2 y}, q=1, \ldots, N_{2 z},
$$

where $l, p, q$ represent the order of the $n$ 2-th small grid in $x, y, z$ directions respectively. We select $M 1$ big grid randomly, put the reference station at the center of each selected grid, and determine the corresponding coordinate of each reference station.

The fine of the meshing depends on the desired positioning accuracy. The higher of the positioning accuracy required, the finer of the meshing. However, with the finer of the meshing, more storage space and long positioning time are needed. In practice, we need to make a balance of the positioning accuracy, the complexity of locating and the positioning time according to the actual situation.

(2) Establish over-complete dictionary $\Phi$.

First of all, transmit the $60 \mathrm{GHz}$ pulse signals at the each large grid node, receive signal at the each reference station to obtain the propagation delay $\tau_{m 1, n 1}$ (where $1 \leq m 1 \leq M 1,1 \leq n 1 \leq N 1$ ), then calculate the actual distance $d_{m 1, n 1}$ based on TOA between the $m 1$-th reference station and the $n 1$-th grid node on basis of $d_{m 1, n 1}=c * \tau_{m 1, n 1}$. Thus, the actual measured distance between each reference station and each large grid node constitutes the distance feature vector $D_{n 1}=\left[d_{1, n 1}, d_{2, n 1}, \ldots \ldots, d_{M 1, n 1}\right]^{T}$. Establish an over-complete dictionary $F$ in which $D_{n 1}$ is correspond with the location of the large grid node $n 1$ as shown in Table 1, where $F_{n 1}$ is a sub-over-complete dictionary established for the $n 1$-th large node in the same way as shown in Table 2. $F_{n 1}$ is comprised of the position of each $\operatorname{small} \operatorname{grid}\left(x_{n 2}, y_{n 2}, z_{n 2}\right),($ where, $1 \leq n 2 \leq N 2)$ and the distance feature vectors $D_{n 2}=\left[d_{1, n 2}, d_{2, n 2}, \ldots \ldots, d_{M 1, n 2}\right]^{T}$ concluding the distance between the n2-th small grid node in the $n 1$-th large grid node and the each reference station.

Table 1: The composition of over-complete dictionary $F$

\begin{tabular}{|c|c|c|c|}
\hline Large grid node & $D_{n 1}$ & $\left(x_{n 1}, y_{n 1}, z_{n 1}\right)$ & sub-over-complete dictionary \\
\hline Large grid 1 & $\left(d_{1,1}, d_{2,1}, \cdots, d_{M 1,1}\right)^{T}$ & $\left(x_{1}, y_{1}, z_{1}\right)$ & $F_{1}$ \\
\hline Large grid 2 & $\left(d_{1,2}, d_{2,2}, \cdots, d_{M 1,2}\right)^{T}$ & $\left(x_{2}, y_{2}, z_{2}\right)$ & $F_{2}$ \\
\hline$\vdots$ & $\vdots$ & $\vdots$ & $\vdots$ \\
\hline Large grid n1 & $\left(d_{1, n 1}, d_{2, n 1}, \cdots, d_{M 1, n 1}\right)^{T}$ & $\left(x_{n 1}, y_{n 1}, z_{n 1}\right)$ & $F_{n 1}$ \\
\hline$\vdots$ & $\vdots$ & $\vdots$ & $\vdots$ \\
\hline Large grid N1 & $\left(d_{1, N 1}, d_{2, N 1}, \cdots, d_{M 1, N 1}\right)^{T}$ & $\left(x_{N 1}, y_{N 1}, z_{N 1}\right)$ & $F_{N 1}$ \\
\hline
\end{tabular}


Table 2: The composition of sub-over-complete dictionary $F_{n 1}$

\begin{tabular}{|c|c|c|}
\hline small grid node & $D_{n 2}$ & $\left(x_{n 2}, y_{n 2}, z_{n 2}\right)$ \\
\hline small grid 1 & $\left(d_{1,1}, d_{2,1}, \cdots, d_{M 1,1}\right)^{T}$ & $\left(x_{1}, y_{1}, z_{1}\right)$ \\
\hline small grid 2 & $\left(d_{1,2}, d_{2,2}, \cdots, d_{M 1,2}\right)^{T}$ & $\left(x_{2}, y_{2}, z_{2}\right)$ \\
\hline$\vdots$ & $\vdots$ & $\vdots$ \\
\hline small grid n2 & $\left(d_{1, n 2}, d_{2, n 2}, \cdots, d_{M 1, n 2}\right)^{T}$ & $\left(x_{n 2}, y_{n 2}, z_{n 2}\right)$ \\
\hline$\vdots$ & $\vdots$ & $\vdots$ \\
\hline small grid N2 & $\left(d_{1, N 2}, d_{2, N 2}, \cdots, d_{M 1, N 2}\right)^{T}$ & $\left(x_{N 2}, y_{N 2}, z_{N 2}\right)$ \\
\hline
\end{tabular}

Establish a measurement matrix $\Phi_{1}=\left[D_{1}, D_{2}, \ldots, D_{n 1}, \ldots, D_{N 1}\right]$ for coarse positioning according to over-complete dictionary $F$ and make an orthogonal operation to the measurement matrix. In the same way, establish a measurement matrix $\Phi_{n 1}=\left[D_{1}, D_{2}, \ldots, D_{n 2}, \ldots, D_{N 2}\right]$ for precise positioning according to sub-over-complete dictionary $F_{n 1}$, but don't make any treatment. Store the measurement matrix $\Phi_{1}$ and $\Phi_{n 1}(1 \leq n 1 \leq N 1)$ in the computing center for online positioning stage.

\subsubsection{The online stage}

Online positioning stage is divided into two process: coarse positioning process and fine positioning process. Fine positioning uses the selection mechanism of the reference station to complete the localization based on the result of the coarse positioning.

1. Coarse positioning process

(1) Select the target node and establish the model of sparse localization

In this paper, the target node can be selected in anywhere of the located area, instead of being limited to the center of the large grid. Select a target node randomly and set the actual position of the target node is $(x, y, z)$. The center coordinates of the large grid in which the target node locate is $\left(x_{n}, y_{n}, z_{n}\right)$. The index of the large grid is $N x$. The position of the target node in the area to be located is described as a sparse vector $X_{1}=[0, \ldots, 0,1,0, \ldots, 0]^{T}$, where the $N x$ corresponds the index of 1 and the position of the other grids are represented by 0 .

(2) Positioning though the reconstruction algorithm

Firstly, the target node transmits $60 \mathrm{GHz}$ pulse signal to each reference station. The actual distance $y_{m}$ (where, $1 \leq m \leq M 1$ ) between the target node and each reference station is measured based on TOA and aggregated to the computing center expressed as measurement column vector $Y_{1}=\left[\begin{array}{llll}y_{1}, & y_{2}, & \cdots & y_{M 1}\end{array}\right]$. Combined with the measurement matrix $\Phi_{1}$, the mathematical model of the positioning process based on CS can be represented by Equation (3.3):

$$
\hat{X}_{1}=\underset{X_{1}}{\arg \min }\left\|X_{1}\right\|_{l_{1}} \text { s.t. } Y_{1}=\Phi_{1} X_{1} .
$$

After reconstructing the sparse vector $\hat{X}_{1}$, we take last $1=\max \left(\hat{X}_{1}\right)$ as the result of the rough positioning. The index of the large grid in which the target located is last1.

2. Fine positioning process

(1) Establish the reference station selection matrix $S$

According to the large grid index last 1 where the target node located determined in the coarse positioning process, identify the distance feature vector $D_{\text {last } 1}=\left[d_{1, \text { last } 1}, d_{2, \text { last } 1}, \ldots \ldots, d_{M 1, \text { last } 1}\right]^{T}$ corresponding to last 1 from the over-complete dictionary.

Determine the number of reference station M2 (M2 $\leq M 1)$ required in the precise positioning process according to the number of small grid nodes. Create index set $Q$ by choosing the corresponding index of the smallest $M 2$ value in the distance feature vector $D_{l a s t 1}$, and then establish a reference station selection matrix $S \in M 2 \times M 1$ according to the $Q$. Each row of $S$ is a $1 \times M 1$ vector in which the index of the reference station selected is set to 1 , namely $S_{m 2}(m 1)=1$, (where $m 2$ represents the $m 2$-th row of the matrix $S, m 1$ represents the $m 1$-th column of the matrix $S$ ), and the other indexes are set to 0 , that is:

$$
S_{m 2}=[0, \ldots, 0,1,0, \ldots, 0] \forall m 2 \in[1,2, \ldots, M 2] .
$$


(2) Construct the measurement matrix of fine positioning and positioning though the reconstruction algorithm

Assume that the measurement matrix required in fine positioning process is $\Phi_{2}$, then $\Phi_{2}=S \times \Phi_{\text {last } 1}$, and an orthogonal operation is made to the measurement matrix $\Phi_{2}$. The position of target node in the large grid is expressed by a sparse vector $X_{2}=[0, \ldots, 0,1,0, \ldots, 0]^{T}$. The actual measured distance between the target node and the each selected reference station constitutes the measurement vector $Y_{2}=\left[y_{1}, y_{2}, \cdots, y_{M 2}\right]$. Then, the $l_{1}$-minimization is used to complete the localization of the target node. The mathematical model of positioning can be described as Equation (3.5):

$$
\hat{X}_{2}=\underset{X_{2}}{\arg \min }\left\|X_{2}\right\|_{l_{1}} \text { s.t. } Y_{2}=\Phi_{2} X_{2} .
$$

After reconstructing the sparse vector $\hat{X}_{2}$, we take last $2=\max \left(\hat{X}_{2}\right)$ as the final positioning result of the target node. last 2 is the index of the small grid node of the target node and the corresponding estimated value of the target node is $(\hat{x}, \hat{y}, \hat{z})$.

(3) Assess the positioning performance

In this paper, the mean square error is used to evaluate the performance of the positioning result, therefore, the positioning error can be expressed as:

$$
e_{M S E}=\sqrt{(x-\hat{x})^{2}+(y-\hat{y})^{2}+(z-\hat{z})^{2}}
$$

The program flow chart of the single target positioning is shown in Fig. 3 .

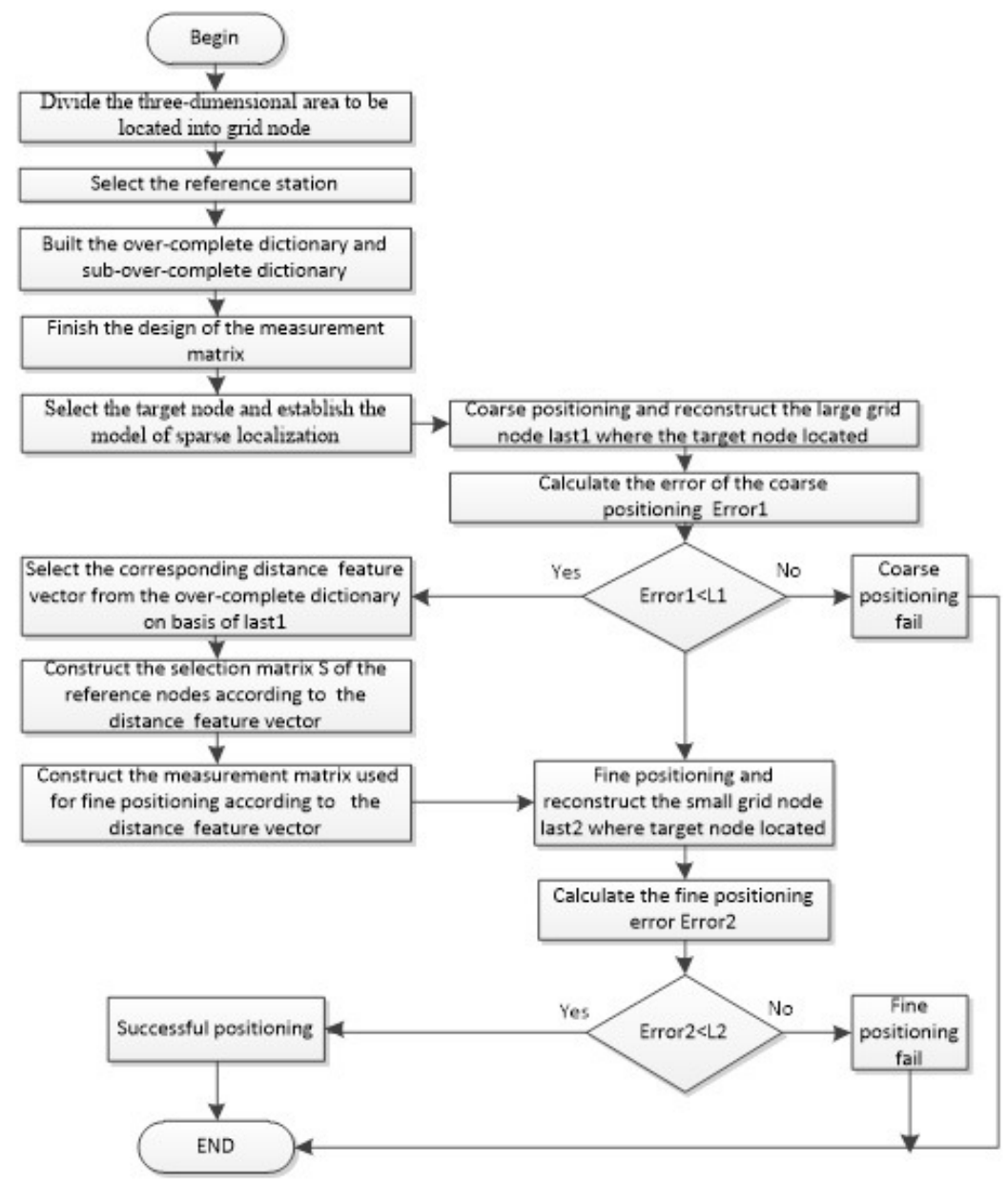

Figure 3: The flow chart of single target positioning. 


\subsection{Multi-target positioning using 60GHz pulse}

The common used positioning algorithms such as the geometric positioning algorithm and the fingerprinting positioning algorithm cannot locate multi-target at the same time. The indoor precise positioning algorithm using $60 \mathrm{GHz}$ pulse based on CS proposed in this paper can realize multi-target localization. The multi-target positioning process includes the offline stage and the online stage. The offline stage is identical with the single target positioning. Then, the different points from the single target positioning at the online stage is mainly introduced. The muli-target positioning program flow chart is shown in Fig. 4.

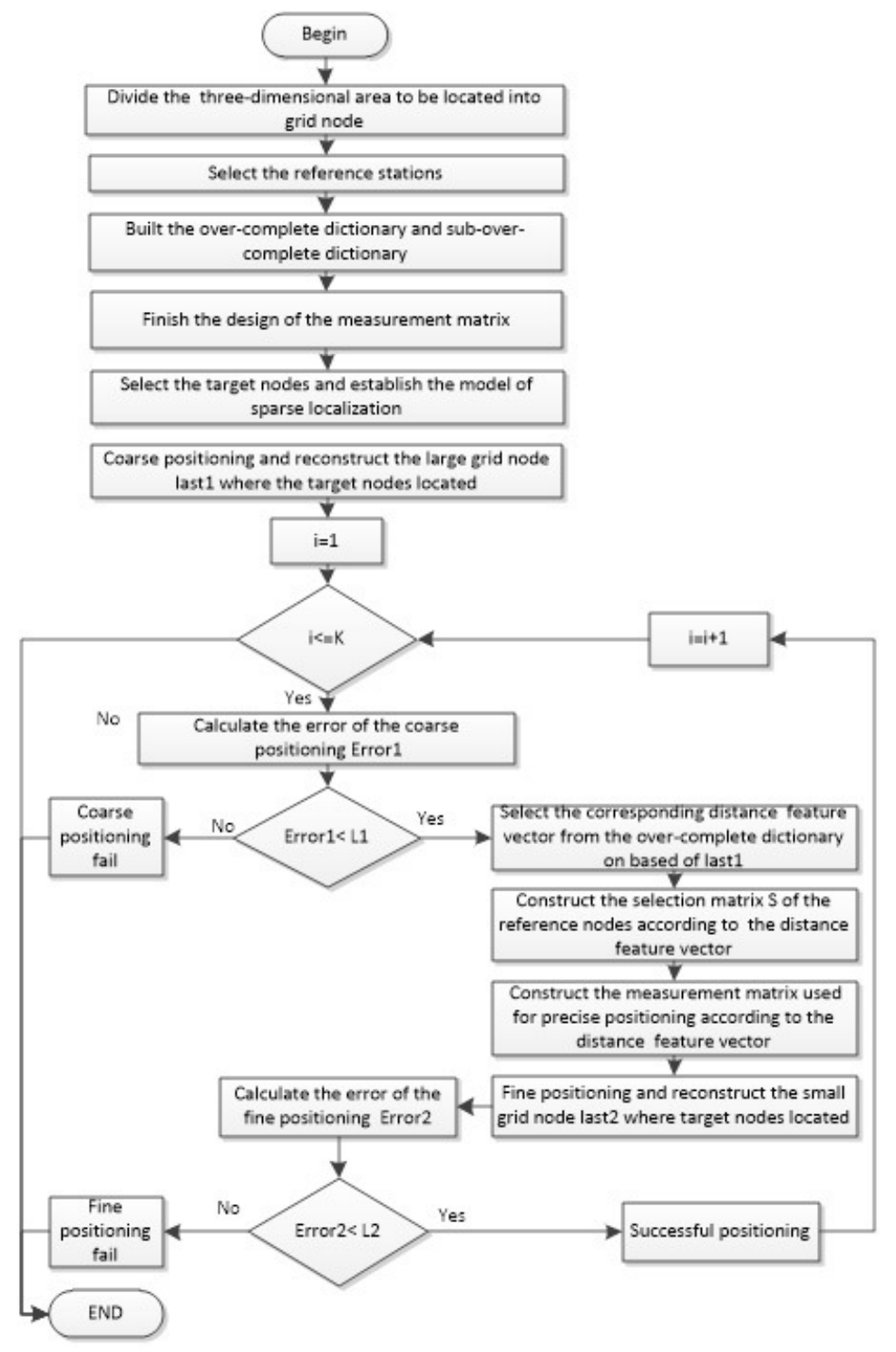

Figure 4: The flow chart of multi-target positioning.

1. Coarse positioning process

(1) Select the target nodes and establish the model of sparse localization

$K$ target nodes are selected at the same time during multi-target positioning. The locations of the target nodes in the area to be located is represented by a $K$-sparse vector, where 1 corresponds to the indexes of the target nodes and 0 represents the other positions.

(2) Positioning though the reconstruction algorithm

During the reconstruction of the positioning process, the $m$-th row $y_{m}$ of the measurement vector $Y 1$ is the sum of the actual measured distance $y_{m k}$ (where $1 \leq m \leq M 1,1 \leq k \leq K$ ) between the $m$-th reference 
station and each target nodes. That is:

$$
Y 1=\left[y_{11}+y_{12}+\ldots+y_{1 K}, y_{21}+y_{22}+\ldots+y_{2 K}, \ldots \ldots, y_{M 1}+y_{M 2}+\ldots+y_{M K}\right]^{T} .
$$

After reconstructing the sparse vector $\hat{X}_{1}$, the corresponding index of the $K$ maximum values of $\hat{X}_{1}$ are considered as the results of the target nodes in coarse positioning process.

2. Fine positioning process

In the process of the multi-target positioning, the positioning computing center has to make a fine positioning for each target node depending on the results of the coarse positioning which is the same with the single target positioning process.

\section{The results and analysis of simulation}

In this part, simulations is conducted to verify the positioning performance of the indoor precise positioning algorithm using $60 \mathrm{GHz}$ pulse based on CS proposed in this paper in NLOS environment. MATLAB is used to perform all simulations. The area to be located is assumed as a $5 m \times 5 m \times 5 m$ square region. The $60 \mathrm{GHz}$ pulse signal is used and PPM-TH modulation scheme is chosen. The typical $60 \mathrm{GHz}$ channel model IEEE802.15.3c is used and the channel model CM2 to simulate the living environment indoor in NLOS is selected. The signal to noise ratio SNR is set to 20dB. The specific simulation process is as follows:

\subsection{The simulation of the 60GHz pulse waveform}

The method of generating $60 \mathrm{GHz}$ pulse is divided into no-carrier pulse program and carrier pulse program. The carrier pulse program is a pulse design method that use the sine or cosine carriers whose center frequency in $60 \mathrm{GHz}$ to move the spectrum of the pulse-based baseband signal to $60 \mathrm{GHz}$ band. In this paper, the carrier pulse program is adopted and the triangular pulse is employed as baseband signal. Therefore, the pulse expression is shown as Equation (4.1):

$$
f(t)=\left(1-\frac{2}{T_{P}} *\left|t-\frac{1}{2 T_{p}}\right|\right) * \cos (2 \pi * f c * t),
$$

Where $T_{p}=0.32 e^{-9} s$ is the duration of the pulse, and $f_{c}=60.5 e^{9} \mathrm{~Hz}$ is the carrier frequency. The waveform in the time domain is shown in Fig. 5 after normalized.

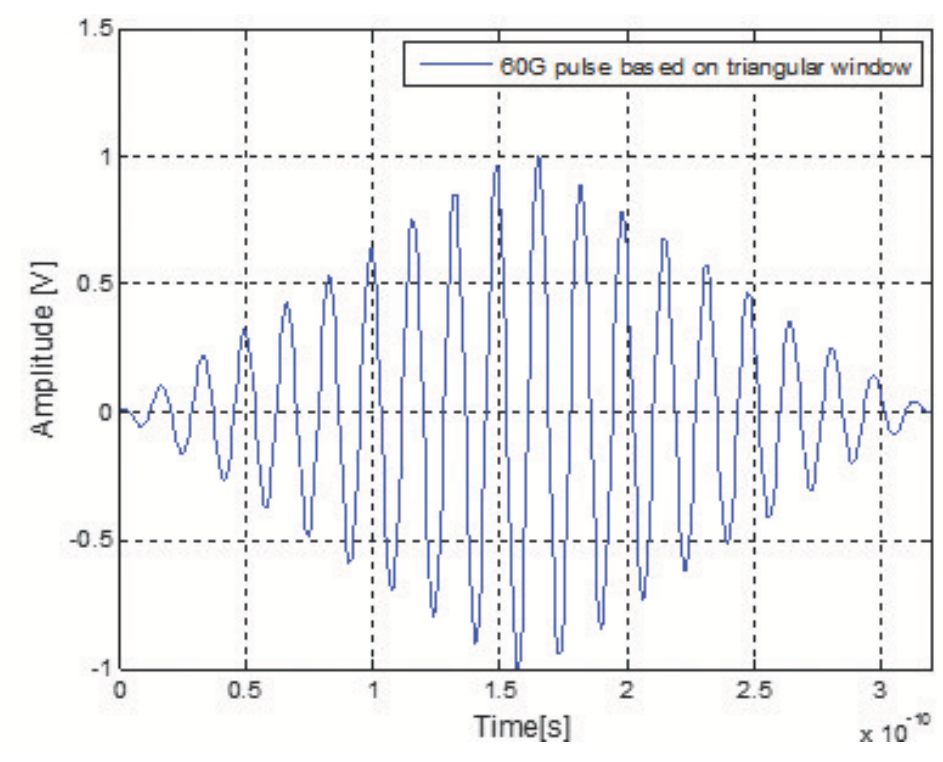

Figure 5: The $60 \mathrm{GHz}$ triangular pulse. 


\subsection{Single target positioning}

Stimulate the process of single target positioning firstly. The simulation results are as follows:

\subsubsection{Coarse positioning process}

The side of the large grid is set to $0.5 \mathrm{~m}$. 30 reference stations are randomly selected and the target node is chosen at random with coordinate $(4.5649,2.9552,2.9226)$. The index of the target node $N x=560$. The coarse positioning result is shown in Fig. 6. The coarse positioning coordinate of the target node is (4.7500, $2.7500,2.7500)$. The located index of the target node last $1=560$. The coarse positioning error $\operatorname{Err} N x 1=$ 0.3258. As the simulation results shown, the coarse positioning can locate the large grid of the target node accurately.

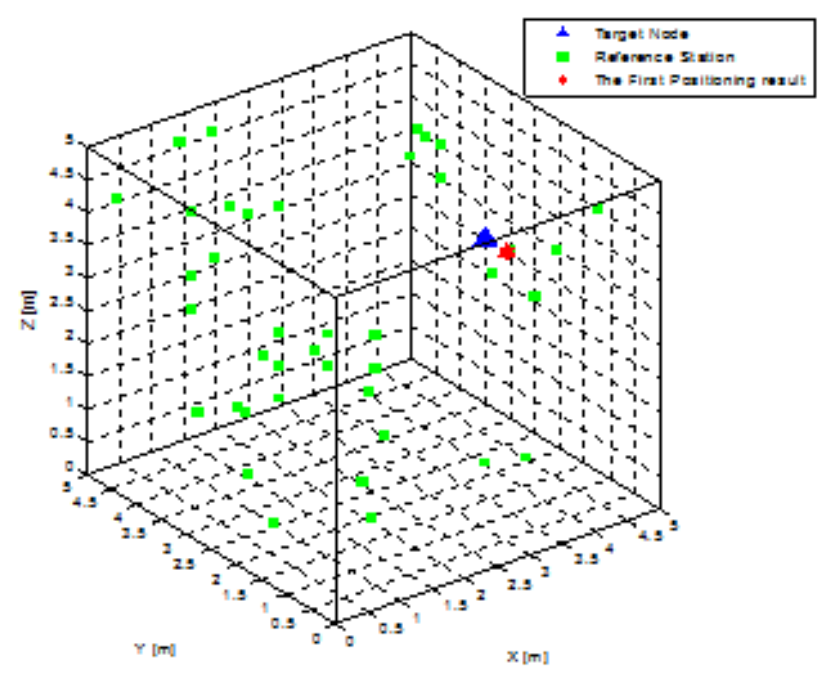

Figure 6: The coarse positioning result.

\subsubsection{Fine positioning process}

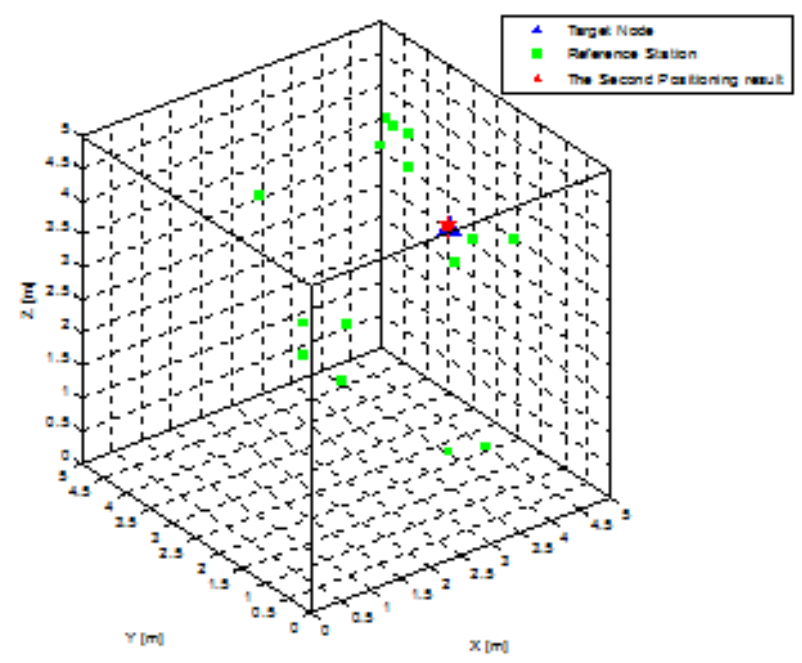

Figure 7: The fine positioning result. 
The side of the small grid is set to $0.1 \mathrm{~m} .15$ reference stations are selected for fine positioning process according to the reference station selection mechanism. The fine positioning method is used to complete the positioning process and the fine positioning result is shown in Fig. 7. The fine positioning coordinate of the target node is $(4.5500,2.9500,2.9500)$. The fine positioning error $\operatorname{Err} N x 2=0.0316$. The amplification of the results of the two positioning process to large grid respectively is shown in Fig. 8. After amplification, it can be seen that the error is very large after the coarse positioning process, but it is small after the fine positioning process. Thus, through twice positioning process the algorithm can achieve centimeter-level indoors positioning accuracy in NLOS.

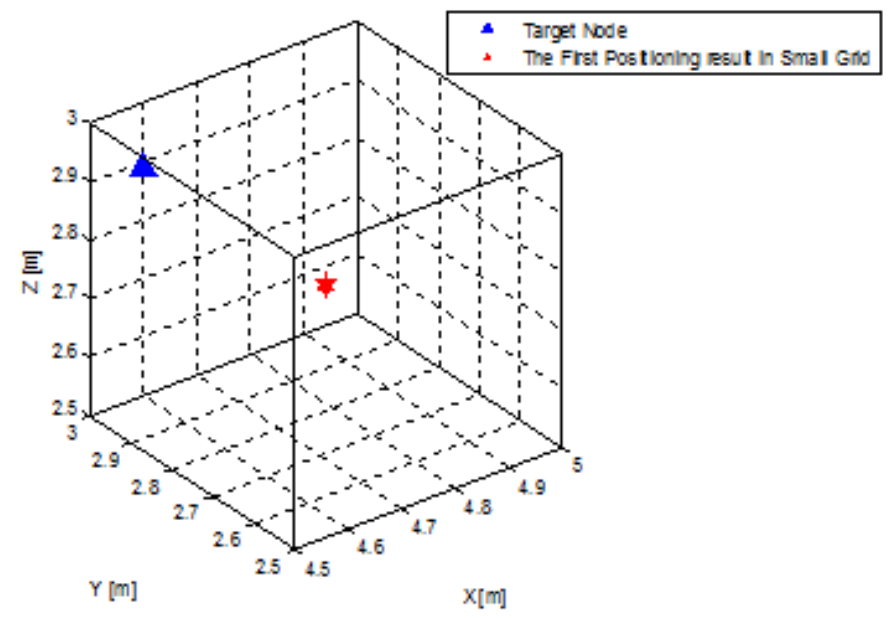

(a) The first positioning result in small grid

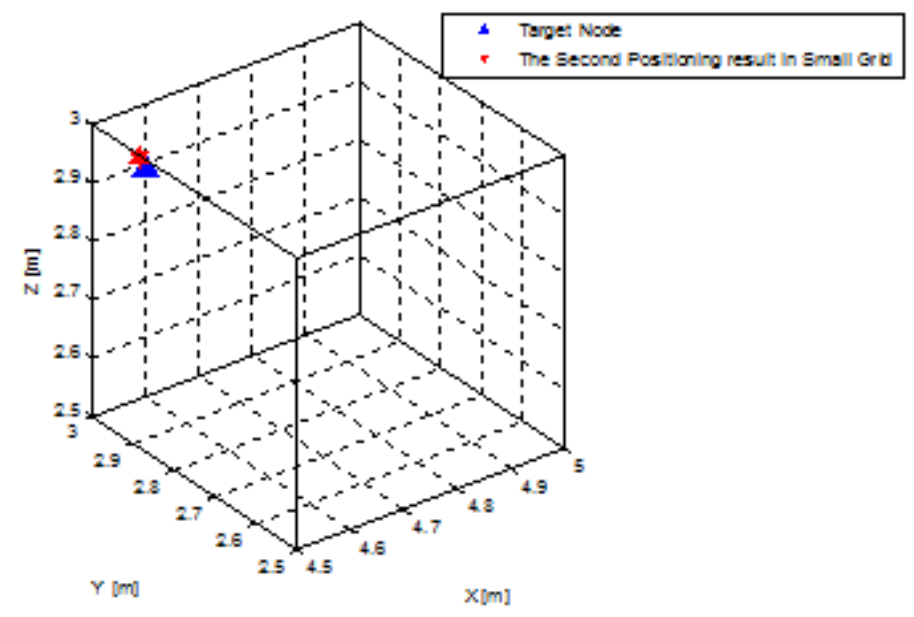

(b) The second positioning result in small grid

Figure 8: The statistics and analysis of simulation results.

\subsubsection{The statistics and analysis of simulation results}

Table 3 shows the 10 times simulation statistical results of the positioning algorithm under the same conditions. The average error of the 10 times localization result is $0.0736 \mathrm{~m}$. Moreover, the positioning accuracy of seven times among them is less $0.1 \mathrm{~m}$. From the simulation results, it can be obtained that the localization algorithm proposed in this paper can realize centimeter-level indoor positioning accuracy in NLOS in high probability. 
Table 3: The statistic results of 10 times single target positioning

\begin{tabular}{|l|l|l|l|l|}
\hline $\begin{array}{l}\text { Actual coordinates of } \\
\text { the target point }\end{array}$ & $\begin{array}{l}\text { Coarse positioning } \\
\text { coordinates }\end{array}$ & $\begin{array}{l}\text { Fine location coordi- } \\
\text { nates }\end{array}$ & $\begin{array}{l}\text { Coarse } \\
\text { loca- } \\
\text { tion } \\
\text { error }\end{array}$ & $\begin{array}{l}\text { Fine } \\
\text { loca- } \\
\text { tion } \\
\text { error }\end{array}$ \\
\hline$(1.4440,4.3355,1.0868)$ & $(1.2500,4.2500,1.2500)$ & $(1.4500,4.3500,1.0500)$ & 0.2675 & 0.0401 \\
\hline$(2.9042,0.7055,1.6635)$ & $(2.7500,0.7500,1.7500)$ & $(2.8500,0.6500,1.6500)$ & 0.1823 & 0.0787 \\
\hline$(2.9839,0.4876,0.1631)$ & $(2.7500,0.2500,0.2500)$ & $(2.9500,0.4500,0.0500)$ & 0.3446 & 0.1239 \\
\hline$(3.9438,2.6540,0.2486)$ & $(3.7500,2.7500,0.2500)$ & $(3.9500,2.6500,0.1500)$ & 0.2163 & 0.0988 \\
\hline$(2.1365,2.9982,0.3402)$ & $(2.2500,2.7500,0.2500)$ & $(2.1500,2.9500,0.2500)$ & 0.2874 & 0.1031 \\
\hline$(0.9774,2.1287,2.4179)$ & $(0.7500,2.2500,2.2500)$ & $(0.9500,2.1500,2.4500)$ & 0.3076 & 0.0473 \\
\hline$(0.1956,2.5303,2.5733)$ & $(0.2500,2.7500,2.7500)$ & $(0.1500,2.5500,2.5500)$ & 0.2871 & 0.0548 \\
\hline$(2.8464,3.8044,0.9607)$ & $(2.7500,3.7500,0.7500)$ & $(2.9500,3.8500,0.9500)$ & 0.2380 & 0.1137 \\
\hline$(2.3640,2.2684,0.7006)$ & $(2.2500,2.2500,0.7500)$ & $(2.3500,2.2500,0.6500)$ & 0.1257 & 0.0556 \\
\hline$(2.6520,1.6646,2.3369)$ & $(2.7500,1.7500,2.2500)$ & $(2.6500,1.6500,2.3500)$ & 0.1564 & 0.0198 \\
\hline
\end{tabular}

\subsubsection{The comparison of positioning performance}

This article makes a comparison of the positioning performance between the $60 \mathrm{GHz}$ pulse indoor precise positioning algorithm based on CS and the geometric positioning algorithm based on TOA in which the coordinate of the target node is determined by using the least squares estimation (LSE). Each location algorithm runs 10000 times under the same conditions respectively. The statistical errors of the two localization algorithms are shown in Fig. 9 and Fig. 10. As it can be seen from the statistical results that the error of the $60 \mathrm{GHz}$ pulse indoor precise positioning algorithm based on CS mainly focuses on the range of $0-0.15 \mathrm{~m}$, and more than 70 percent of it are concentrated in the scope less than $0.1 \mathrm{~m}$. In comparison, more than 80 percent of the error generated by the $60 \mathrm{GHz}$ pulse geometric positioning algorithm based on TOA is located in the range of $0.1-0.25 \mathrm{~m}$. Therefore, compared with the TOA based $60 \mathrm{GHz}$ pulse geometric positioning algorithm, the CS based $60 \mathrm{GHz}$ pulse indoor precise positioning algorithm can realizes the centimeter-level indoor positioning accuracy in NLOS with high probability.

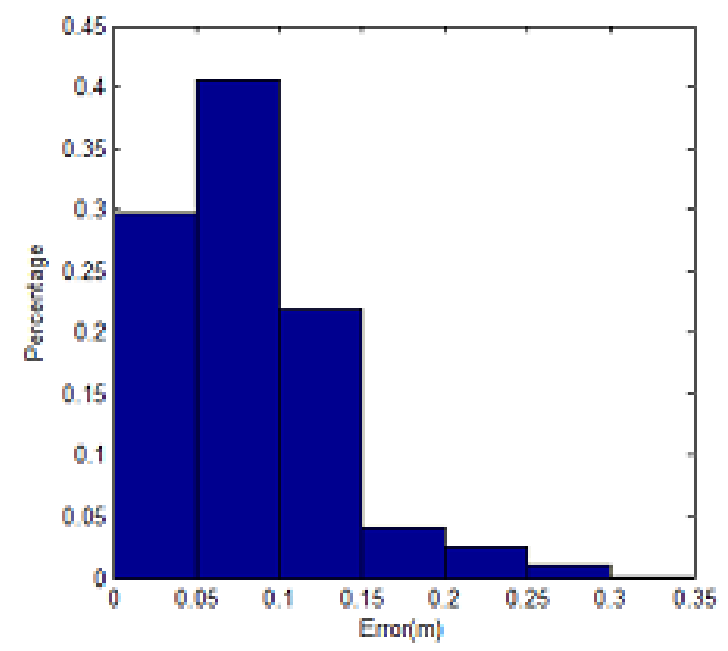

Figure 9: The distribution of the error generated by $60 \mathrm{GHz}$ pulse indoor precise positioning algorithm based on CS in NLOS. 


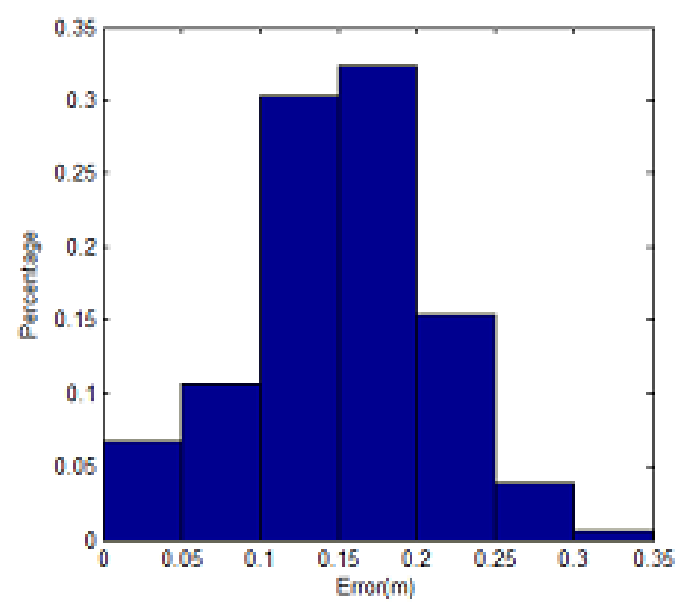

Figure 10: The distribution of the error generated by $60 \mathrm{GHz}$ pulse geometric positioning algorithm based on TOA in NLOS.

\subsection{Multi-target positioning}

\subsubsection{The coarse positioning process of the multi-target}

The side of the large grid is set to $0.5 \mathrm{~m}$, that means $N_{1}=1000.40$ reference stations are selected randomly and three target nodes are chosen freely with coordinates $\{(1.6473,1.5405,0.3796),(4.7592,3.0352,3.4314)$, $(1.7715,3.4217,4.1935)\}$. The indexes of the target nodes $N x=\{34,670,864\}$. Positioning the $\mathrm{K}$ target nodes at the same time according to the principle of the multi-target positioning. The coarse positioning results is shown as Fig. 11. The coarse positioning coordinates of the target nodes are $\{(1.7500,1.7500$, $0.2500),(4.7500,3.2500,3.2500),(1.7500,3.2500,4.2500)\}$. The located indexes of the target nodes last1 = $\{34,670,864\}$. The coarse positioning errors $\operatorname{Err} N x 1=[0.2669,0.2813,0.1821]$. As the simulation results shown, the coarse positioning can locate the large grids of the each target nodes accurately which is the precondition of fine positioning process.

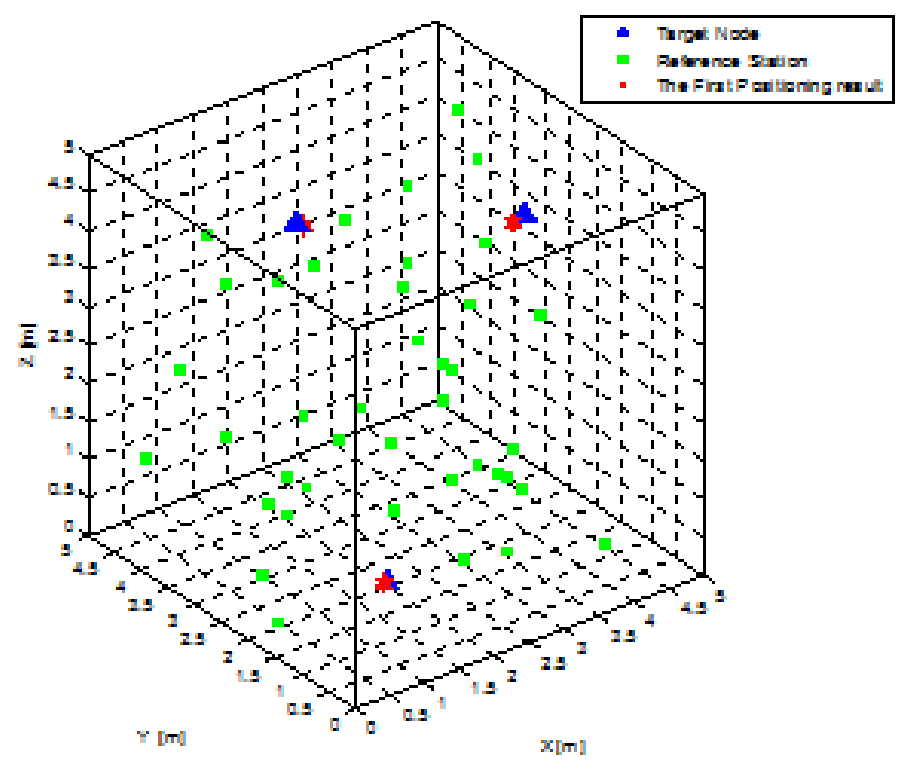

Figure 11: The coarse positioning results of multi-target . 


\subsubsection{The fine positioning process of the multi-target}

The side of the small grid is set to $0.1 \mathrm{~m}$, that mean $N_{2}=125.15$ reference stations are selected at random for fine positioning process according to the reference station selection mechanism. The fine positioning result is shown in Fig. 12 when the fine positioning method is used to complete the positioning process. The fine positioning coordinates of the target nodes are $\{(1.6500,1.5500,0.4500),(4.8500,3.0500,3.4500)$, $(1.7500,3.4500,4.1500)\}$. The fine positioning errors $\operatorname{Err} N x 2=[0.0711,0.0938,0.0562]$. It can be seen from the simulation results that the fine positioning process can achieve centimeter-level indoors positioning accuracy using $60 \mathrm{GHz}$ pulse in NLOS environment.

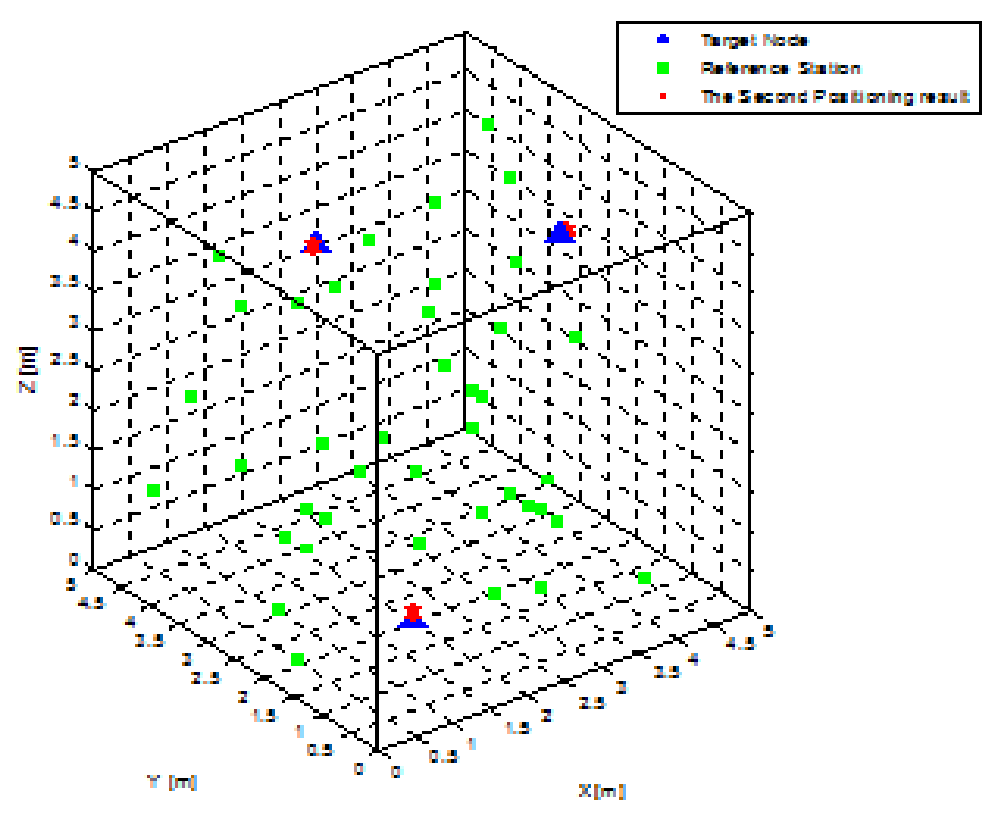

Figure 12: The fine positioning results of multi-target .

\subsubsection{The statistics and analysis of the repeated simulation results}

Table 4 shows 6 times simulations statistical results of the positioning algorithm under the same conditions. The average error of the 6 times localization result is $0.0869 \mathrm{~m}$. What's more, the positioning error of 13 targets is under $0.1 \mathrm{~m}$ in 18 single targets positioning result. From the simulation results, it can be obtained that the localization algorithm proposed in this paper can locate several target at the same time and realizes centimeter-level indoor positioning accuracy in NLOS with high probability.

\subsection{The analysis of error}

Through a comprehensive analysis of the positioning algorithm in this paper, the reason resulting the positioning errors are mainly from the following aspects:

(1) The accuracy of the meshing play a decisive role in positioning accuracy and complexity. The finer of the meshing, the higher of the positioning accuracy, however, it will lead to the increase of the computational complexity at the same time. Therefore, in designing the accuracy of the grid we have to weigh both the positioning accuracy, the complexity and make a compromise.

(2) In this paper, the position of the target nodes are selected randomly, therefore, the target nodes can be located in anywhere of the located area. However, the positioning results can only be accurate to the center of a small grid. Thus, the positioning errors exist without a doubt and cannot be reduced to zero. 
Table 4: The statistic results of 6 times multi- target positioning

\begin{tabular}{|c|c|c|c|c|c|c|c|}
\hline Times & & $\begin{array}{l}\text { ual coordi- } \\
\text { es of the tar- } \\
\text { points }\end{array}$ & $\begin{array}{l}\text { Coarse positioning } \\
\text { coordinates }\end{array}$ & $\begin{array}{l}\text { Fine positioning } \\
\text { coordinates }\end{array}$ & $\begin{array}{l}\text { Coarse } \\
\text { position- } \\
\text { ing } \\
\text { error }\end{array}$ & $\begin{array}{l}\text { Fine } \\
\text { posi- } \\
\text { tion- } \\
\text { ing } \\
\text { error }\end{array}$ & $\begin{array}{l}\text { Average } \\
\text { error }\end{array}$ \\
\hline \multirow{3}{*}{$\begin{array}{l}\text { First } \\
\text { time }\end{array}$} & 1 & $(1.0604,2.8210,2.5415)$ & $(1.2500,2.7500,2.7500)$ & $(1.0500,2.9500,2.5500)$ & 0.2906 & 0.1297 & \multirow{3}{*}{0.1082} \\
\hline & 2 & $(4.9333,4.6012,1.7012)$ & $(4.7500,4.7500,1.7500)$ & $(4.9500,4.5500,1.6500)$ & 0.2411 & 0.0743 & \\
\hline & 3 & $(1.8950,4.3427,3.5428)$ & $(1.7500,4.2500,3.7500)$ & $(1.9500,4.4500,3.5500)$ & 0.2693 & 0.1207 & \\
\hline \multirow{3}{*}{$\begin{array}{l}\text { Second } \\
\text { time }\end{array}$} & 1 & $(3.0842,0.9742,3.6138)$ & $(3.2500,0.7500,3.7500)$ & $(3.0500,0.9500,3.5500)$ & 0.3104 & 0.0763 & \multirow{3}{*}{0.0612} \\
\hline & 2 & $(1.6186,3.5435,1.4029)$ & $(1.7500,3.7500,1.2500)$ & $(1.6500,3.5500,1.4500)$ & 0.2886 & 0.0570 & \\
\hline & 3 & $(3.9030,3.1545,2.1672)$ & $(3.7500,3.2500,2.2500)$ & $(3.9500,3.1500,2.1500)$ & 0.1985 & 0.0502 & \\
\hline \multirow{3}{*}{$\begin{array}{l}\text { Third } \\
\text { time }\end{array}$} & 1 & $(3.1332,4.9712,0.8348)$ & $(3.2500,4.7500,0.7500)$ & $(3.0500,4.9500,0.9500)$ & 0.2641 & 0.1437 & \multirow{3}{*}{0.1110} \\
\hline & 2 & $(0.0623,2.3813,2.8275)$ & $(0.2500,2.2500,2.7500)$ & $(0.0500,2.2500,2.8500)$ & 0.2418 & 0.1338 & \\
\hline & 3 & $(2.2426,1.6944,3.6176)$ & $(2.2500,1.7500,3.7500)$ & $(2.2500,1.6500,3.6500)$ & 0.1437 & 0.0555 & \\
\hline \multirow{3}{*}{$\begin{array}{c}\text { Fourth } \\
\text { time }\end{array}$} & 1 & $(4.1277,4.7331,4.3926)$ & $(4.1500,4.7500,4.2500)$ & $(4.1500,4.7500,4.4500)$ & 0.1453 & 0.0639 & \multirow{3}{*}{0.0669} \\
\hline & 2 & $(2.2461,0.4017,1.8471)$ & $(2.2500,0.2500,1.7500)$ & $(2.2500,0.4500,1.8500)$ & 0.1802 & 0.0485 & \\
\hline & 3 & $(4.3663,3.9237,1.1969)$ & $(4.2500,3.7500,1.2500)$ & $(4.3500,3.8500,1.1500)$ & 0.2159 & 0.0884 & \\
\hline \multirow{3}{*}{$\begin{array}{r}\text { Fifth } \\
\text { time }\end{array}$} & 1 & $(4.2327,3.9642,1.0911)$ & $(4.2500,3.7500,1.2500)$ & $(4.2500,3.9500,1.1500)$ & 0.2673 & 0.0623 & \multirow{3}{*}{0.0602} \\
\hline & 2 & $(3.0447,2.9568,0.7149)$ & $(3.2500,2.7500,0.7500)$ & $(3.0500,2.9500,0.7500)$ & 0.2935 & 0.0361 & \\
\hline & 3 & $(4.5964,1.9380,2.6887)$ & $(4.7500,1.7500,2.7500)$ & $(4.6500,1.9500,2.7500)$ & 0.2504 & 0.0823 & \\
\hline \multirow{3}{*}{$\begin{array}{r}\text { Sixth } \\
\text { time }\end{array}$} & 1 & $(4.5972,4.6751,3.8621)$ & $(4.7500,4.7500,3.7500)$ & $(4.5500,4.6500,3.7500)$ & 0.2038 & 0.1241 & \multirow{3}{*}{0.1136} \\
\hline & 2 & $(4.5787,4.6020,1.2605)$ & $(4.7500,4,7500,1.2500)$ & $(4.5500,4.5500,1.3500)$ & 0.2266 & 0.1074 & \\
\hline & 3 & $(0.9846,3.1456,3.0900)$ & $(0.7500,3.2500,3.2500)$ & $(0.9500,3.0500,3.0500)$ & 0.3025 & 0.1093 & \\
\hline
\end{tabular}

\section{Conclusion}

This paper presents an indoor precise positioning algorithm using $60 \mathrm{GHz}$ pulse based on CS. The algorithm converts the problems of indoor accurate positioning into the problems of reconstruction in CS through translating the position of the target in area to be located into a sparse vector, completing the design of the over-completed dictionary based on TOA and reconstructing the location of the target nodes by $l_{1}$-minimization to complete the positioning process. The algorithm divides the positioning into coarse positioning process and fine positioning process which greatly reduces the number of the reference stations needed and lower the cost in ensuring of the positioning accuracy. Moreover, the algorithm uses the reference nodes selection mechanism during the fine positioning process to remove the relatively distant reference stations. These measures improve the positioning accuracy to a large extent and reduce the computational complexity. In addition, the positioning algorithm can not only be used to locate a single target but also multi-target. From theoretical analysis and simulation results we can draw the conclusion that the CS based $60 \mathrm{GHz}$ pulse positioning algorithm makes the realization of the centimeter-level positioning accuracy in indoor NLOS environment which is higher than the $60 \mathrm{GHz}$ geometric positioning algorithm based on TOA in NLOS by comparing the statistical positioning results. This study has an important theoretical significance and practical value.

\section{Acknowledgment}

The authors would like to thank the referees and editors for providing very helpful comments and suggestions.

This project was supported by the Key Laboratory of Universal Wireless Communications (Beijing University of Posts and Telecommunications), Ministry of Education, P. R. China (No. KFKT-2013102), the National Natural Science Foundation of China (No. 61304222), the China Postdoctoral Science Foundation (No. 2014M551905), and the Open Research Fund from Shandong provincial Key Laboratory of Computer Network (No. SDKLCN-2015-04). 


\section{References}

[1] R. G. Baraniuk, Compressive sensing, IEEE Signal Proces. Mag., 24 (2007), 118-121. 2.2

[2] T. Baykas, C. S. Sum, Z. Lan, J. Wang, M. A. Rahman, H. Harada, S. Kato, IEEE 802.15.3c: the first IEEE wireless standard for data rates over $1 \mathrm{~Gb} / \mathrm{s}$, IEEE Commun. Mag., 49 (2011), 114-121. 1

[3] E. J. Candes, J. Romberg, Sparity and incoherence in compressive sensing, Inverse Prob., 23 (2007), 969-985. $2.1,2.2$

[4] E. Candes, J. Romberg, T. Tao, Robust uncertainty principles: Exact signal reconstruction from highly incomplete frequency information, IEEE Trans. Inform. Theory, 52 (2006), 489-509. 1

[5] E. J. Candes, J. Romberg, T. Tao, Stable signal recovery from incomplete and inaccurate measurement, Commun. Pure Appl. Math., 59 (2006), 1207-1223. 2.1.

[6] S. S. Chen, D. L. Donho, M. A. Saunders, Atomic decomposition by basis pursuit, SIAM Rev., 43 (2001), 33-61. 2.1

[7] D. Donoho, Compressing sensing, IEEE Trans. Inform. Theory, 52 (2006), 1289-1306. 1

[8] C. Feng, S. Valaee, Z. Tan, Multiple target localization using compressive sensing, IEEE Global Commun. Compres., Honolulu, 30 (2009), 1-6. 1 .

[9] C. Gustafson, $60 \mathrm{GHz}$ Wireless Propagation Channels: Characterization, Modeling and Evaluation, Diss. Lund Univ., 2014 (2014). 1

[10] IEEE P802.11.ad/D9.0, Part 11: Wireless LAN Medium Access Control (MAC) and Physical Layer (PHY) Specifications, IEEE, (2012).1

[11] A. Jafari, J. Sarrazin, D. Lautru, A. Benlarbi-Delai, L. Petrillo, P. De Doncker, NLOS Influence on 60 GHz Indoor Localization Based on a New TDOA Extraction Approach/C], EuMC Conference, (2013), 330-333. 1 ]

[12] B. Li, Z. Zhou, W. Zou, X. Sun, G. Du, On the Efficient Beam-Forming Training for 60GHz Wireless Personal Area Networks, IEEE Trans. Wireless Commun., 12 (2013), 504-515. 1

[13] T. Nagayama, S. Takeda, M. Umehira, K. Kagoshima, T. Miyajima, Improving Performance by Countering Human Body Shadowing in 60GHz Band Wireless Systems by Using Two Transmit and Two Receive Antennas, Ieice Trans. Commun., 99 (2016), 422-429. 1 .

[14] S. Qin, Y. D. Zhang, Q. Wu, M. G. Amin, Structure-Aware Bayesian Compressive Sensing for Near-Field Source Localization Based on Sensor-Angle Distributions, Int. J. Antennas \& Propagation, 2015 (2015), 15 pages. 1

[15] T. Redant, T. Ayhan, N. De. Clercq, M. Verhelst, P. Reynaert, W. Dehaene, 20.1 A 40nm CMOS receiver for $60 \mathrm{GHz}$ discrete-carrier indoor localization achieving mm-precision at $4 \mathrm{~m}$ range, IEEE International Solid-state Circuits Conference, (2014), 342-343. 1

[16] S. Saponara, B. Neri, Fully Integrated 60 GHz Transceiver for Wireless HD/WiGig Short-Range Multi-Gbit Connections, Springer International Publishing, (2016), 131-137. 1

[17] P. Smulders, Exploiting the 60GHz band for local wireless multimedia access: prospects and future directions, IEEE Commun. Mag., 40 (2002), 140-147. 1

[18] G. Souliotis, F. Plessas, F. Liakou, M. Birbas, A $90 \mathrm{~nm}$ CMOS 15/60 GHz frequency quadrupler, Int. J. Electron., 100 (2013), 1529-1545. 1

[19] J. Wang, X. Liang, W. Shi, Q. Niu, T. A. Gulliver, X. Liu, Fingerprinting Localization based on 60 GHz Impulse Radio, IEEE Pacific Rim Conference, (2015), 491-495. 1

[20] J. Wang, W. Shi, X. Wang, L. Xu, Q. Niu, Y. Ma, Design and Simulation of High-Precision Position System using 60GHz pulse, J. Circuits Syst. Comput., 25 (2016), in Press. 1

[21] X. Yu, B. Chi, M. Wei, A. Wang, T. Ren, Z. W. Wei, A half rate CDR with DCD cleaning up and quadrature clock calibration for 20Gbps 60GHz communication in 65nm CMOS, Int. J. Health Services, 44 (2013), 962-965. 1

[22] B. Zhang, X. Cheng, N. Zhang, Y. Cui, Y. Li, Q. Liang, Sparse target counting and localization in sensor networks based on compressive sensing, Infocom. IEEE, 2 (2011), 2255-2263. 1

[23] H. Zhang, X. Chu, W. Guo, S. Wang, Coexistence of Wi-Fi and Heterogeneous Small Cell Networks Sharing Unlicensed Spectrum, IEEE Commun. Mag., 53 (2015), 158-164. 1

[24] H. Zhang, C. Jiang, N. C. Beaulieu, X. Chu, X. Wen, M. Tao, Resource Allocation in Spectrum Sharing OFDMA Femtocells with Heterogeneous Services, IEEE Trans. Commun., 62 (2014), 2366-2377. 1

[25] H. Zhang, H. Liu, C. Jiang, X. Chu, A. Nallanathan, X. Wen, A Practical Semi-dynamic Clustering Scheme Using Affinity Propagation in Cooperative Picocells, IEEE Trans. Vehicular Technol., 64 (2015), 4372-4377. 1

[26] G. Zheng, C. Hua, R. Zheng, Q. Wang, Toward Robust Relay Placement in 60GHz mmWave Wireless Personal Area Networks with Directional Antenna, IEEE Trans. Mobile Comput., 2016 (2016), 762-773. 1 